\title{
Behaviors of Simply Supported Cross-Ply Rectangular Symmetric Laminates Plates with Consideration of Prebuckling In-Plane Deformation
}

\author{
Abdelaziz Lairedj ${ }^{1}$, Mokhtar Bouazza ${ }^{2,3 *}$, Khaled Amara ${ }^{3,4}$ and Abdelmadjid Chabani ${ }^{2}$ \\ 1 Department of mechanical Engineering, University of Bechar, Bechar 08000, Algeria \\ 2 Department of Civil Engineering, University of Bechar, Bechar 08000, Algeria \\ ${ }^{3}$ Laboratory of Materials and Hydrology (LMH), University of Sidi Bel Abbes, Sidi Bel Abbes 2200, Algeria \\ ${ }^{4}$ Department of Civil Engineering, University centre of Ain Temouchent, Ain Temouchent 4600, Algeria
}

\begin{abstract}
This paper addresses the elastic buckling of simply supported cross-ply rectangular symmetric laminates with allowance for the effects of prebuckling in-plane deformation and higher-order strain terms (curvature terms), using the first order shear deformation theory. The governing plate equations are derived by considering the stationary condition for the derived energy functional by applying the calculus of variations. The buckling load of symmetric cross-ply laminated rectangular plates, which are subjected to uniaxial compression, biaxial compression, and biaxial compression and tension, are determined whilst the effects of different parameters, the plate aspect ratio, the relative thickness degrees of material orthotropy and numbers of layers are investigated.
\end{abstract}

Keywords: Prebuckling in-plane deformation; Curvature terms; First order shear deformation theory.

\section{Introduction}

Many investigators have studied the post-buckling behaviour of isotropic and orthotropic plates, using different methods, e.g. finite element method, finite difference method, Ritz method, and Galerkin's method.

Buckling of composite plates has been investigated extensively in the monograph edited by Turvey and Marshall [1]. Pagano [2] developed an exact three-dimensional (3-D) elasticity solution for static analysis of rectangular bidirectional composites and sandwich plates. Noor $[3,4]$ presented a solution for stability and vibration of multi-layered composite plates based on 3-D elasticity theory by solving equations with the finite difference method. Marshell et al. [5] presented a theoretical analysis for post-buckling behaviour of thin orthotropic curved panels subjected to central point load and uniform pressure loading. Kim and Voyiadjis [6] studied the nonlinear bending behaviour of moderately thick plates and shell using an eight nodded shell element with six degrees of freedom per node. 
They have taken the geometrical non-linearity arises owing to both the quadratic terms of the Green strain tensor and the kinematic relation themselves. It is limited to geometric imperfections that reduce the buckling capacity. Kang and Leissa [7] extended their analysis to buckling of rectangular thin plates having two opposite edges simply supported and subjected to linearly varying in-plane load.

Reddy and Phan [8] obtained exact buckling loads and natural frequencies of simply supported rectangular plates by using a higher-order shear deformation theory. Bouazza et al. [9, 10] investigated the buckling behavior of functionally graded material plate under different loading conditions based on the based on the classical plate theory.

Bouazza et al. [11, 12] used the first-order shear deformation theory to derive closed-form relations for buckling temperature difference of simply supported moderately thick rectangular power-law functionally graded plates. Matsunaga $[13,14]$ analyzed the buckling of thick elastic plates subjected to in-plane stresses, with particular emphasis on the variation of natural frequencies in preloaded condition. Noor and Burton [15] proposed some FEM buckling solutions for multilayered composite layers subjected to in-plane and shear loadings. Ferreira et al. investigated the buckling behavior of composite shells by means of a layered formulation [16]. The same author has recently applied a meshless method in order to compute the natural frequencies of thick layered plates [17].

In this work, we investigate the elastic buckling behaviour of simply supported crossply rectangular symmetric' laminates based on first order shear deformation theory and and the incremental total potential energy approach. The governing differential equations for this problem have been derived by considering the stationary condition for the derived energy functional by applying the calculus of variations. Using the Navier solution method, the differential equations have been solved analytically and the critical buckling loads presented in closed-form solutions. Analytical expressions are validated with existing results in the literature for some special cases.

\section{Theoretical formulation 2.1 Incremental potential energy}

Let the present configuration of the plate be $C_{1}$, and the next configuration be $\mathrm{C}_{2}$. The incremental total potential energy functional of the plate can be expressed as:

$$
\begin{aligned}
& \Pi=\frac{1}{2} \int_{v} \sigma_{L} \Delta \varepsilon_{L} d V+\int_{v} \tau \Delta \varepsilon_{N} d V- \\
& -\frac{1}{2}\left(\int_{s} \Delta N_{x} u_{0} d S+\int_{s} \Delta N_{y} v_{0} d S\right)
\end{aligned}
$$

Where: $\mathrm{u}_{0}$ and $\mathrm{v}_{0}$, are the displacements in the $\mathrm{x}$ and $\mathrm{y}$ directions at the midplane of the plate when the plate deforms from $\mathrm{C}_{1}$, to $\mathrm{C}_{2} ; V$ is the volume of the plate at $\mathrm{C}_{1} ; \Delta \varepsilon$ is the incremental Green-Lagrange strain tensor from $\mathrm{C}_{1}$ to $\mathrm{C}_{2} ; \Delta N_{x}$ and $\Delta N_{y}$ are the load increments in the $\mathrm{x}$ and $\mathrm{y}$ directions from $\mathrm{C}_{1}$ to $\mathrm{C}_{2} ; \tau$ is the second PiolaKirchhoff stress tensor at $\mathrm{C}_{1}$; and the subscripts $\mathrm{L}$ and $\mathrm{N}$ denote linear and nonlinear components, respectively.

\subsection{Kinematics}

A standard $\mathrm{x}, \mathrm{y}, \mathrm{z}$ Cartesian coordinate system is located at the center of the plate, as illustrated in Fig. 1. The plate thickness and in-plane dimensions are denoted by h, a and b, respectively. In addition the plate is composed of an arbitrary number of layers with arbitrary fiber orientation (denoted by $\theta$ ) in each layer. The Mindlin-type laminated plate equations'for bending and buckling of symmetric, anisotropic laminates are based on the assumed displacement field

$$
\begin{aligned}
& u(x, y, z)=u_{0}(x, y)+z \theta_{x}(x, y) \\
& v(x, y, z)=v_{0}(x, y)+z \theta_{y}(x, y) \\
& w(x, y, z)=w_{0}(x, y)
\end{aligned}
$$

In which $\mathrm{u}, \mathrm{v}$ and $\mathrm{w}$ are the displacement components in $\mathrm{x}, \mathrm{y}$ and $\mathrm{z}$ directions respectively. $\theta_{x}$ and $\theta_{y}$ are also the neutral plate rotations around $\mathrm{y}$ and $\mathrm{x}$ axes respectively. In accordance with the above displacement field, the definitions for $\Delta \varepsilon_{L}$ and $\Delta \varepsilon_{N}$ in the Green-Lagrange straindisplacement relation-ship are [18]: 


$$
\Delta \varepsilon_{L}=\left\{\begin{array}{c}
\varepsilon_{x x L} \\
\varepsilon_{y y L} \\
\gamma_{x y L} \\
\gamma_{y z L} \\
\gamma_{x z L}
\end{array}\right\}=\left\{\begin{array}{c}
\frac{\partial u}{\partial x} \\
\frac{\partial v}{\partial y} \\
\frac{\partial u}{\partial y}+\frac{\partial v}{\partial x} \\
\frac{\partial v}{\partial z}+\frac{\partial w}{\partial y} \\
\frac{\partial u}{\partial z}+\frac{\partial w}{\partial x}
\end{array}\right\}=\left\{\begin{array}{c}
z \frac{\partial \theta_{x}}{\partial x} \\
z \frac{\partial \theta_{y}}{\partial y} \\
z\left(\frac{\partial \theta_{x}}{\partial y}+\frac{\partial \theta_{x}}{\partial x}\right) \\
\theta_{y}+\frac{\partial w_{0}}{\partial y} \\
\theta_{x}+\frac{\partial w_{0}}{\partial x}
\end{array}\right\}
$$

$$
\Delta \varepsilon_{N}=\left\{\begin{array}{c}
\varepsilon_{x x N} \\
\varepsilon_{y y N} \\
\gamma_{x y N} \\
\gamma_{y z N} \\
\gamma_{x z N}
\end{array}\right\}=\left\{\begin{array}{c}
\frac{1}{2}\left[\left(\frac{\partial u}{\partial x}\right)^{2}+\left(\frac{\partial v}{\partial x}\right)^{2}+\left(\frac{\partial w}{\partial x}\right)^{2}\right] \\
\frac{1}{2}\left[\left(\frac{\partial u}{\partial y}\right)^{2}+\left(\frac{\partial v}{\partial y}\right)^{2}+\left(\frac{\partial w}{\partial y}\right)^{2}\right] \\
\frac{\partial u}{\partial x} \frac{\partial u}{\partial y}+\frac{\partial v}{\partial x} \frac{\partial v}{\partial y}+\frac{\partial w}{\partial x} \frac{\partial w}{\partial y} \\
\frac{\partial u}{\partial y} \frac{\partial u}{\partial z}+\frac{\partial v}{\partial y} \frac{\partial v}{\partial z}+\frac{\partial w}{\partial y} \frac{\partial w}{\partial z} \\
\frac{\partial u}{\partial x} \frac{\partial u}{\partial z}+\frac{\partial v}{\partial x} \frac{\partial v}{\partial z}+\frac{\partial w}{\partial x} \frac{\partial w}{\partial z}
\end{array}\right\}=\left\{\begin{array}{c}
\frac{\partial}{2}\left[z^{2}\left(\frac{\partial \theta_{x}}{\partial x}\right)^{2}+z^{2}\left(\frac{\partial \theta_{y}}{\partial x}\right)^{2}+\left(\frac{\partial w_{0}}{\partial x}\right)^{2}\right] \\
\frac{1}{2}\left[z^{2}\left(\frac{\partial \theta_{x}}{\partial y}\right)^{2}+z^{2}\left(\frac{\partial \theta_{y}}{\partial y}\right)^{2}+\left(\frac{\partial w_{0}}{\partial y}\right)^{2}\right] \\
\partial y \\
\frac{\partial \theta_{x}}{\partial x} \frac{\partial \theta_{y}}{\partial y}+\frac{\partial \theta_{0}}{\partial x} \frac{\partial w_{0}}{\partial y} \\
z \theta_{x} \frac{\partial \theta_{x}}{\partial y}+z \theta_{y} \frac{\partial \theta_{y}}{\partial y} \\
z \theta_{x} \frac{\partial \theta_{x}}{\partial x}+z \theta_{y} \frac{\partial \theta_{y}}{\partial x}
\end{array}\right\}
$$

\subsection{Constitutive relations}

Under the assumption that each layer possesses a plane of elastic symmetry parallel to the $\mathrm{x}-\mathrm{y}$ plane, the constitutive equations for a layer can be written as:

$$
\left\{\begin{array}{l}
\sigma_{x} \\
\sigma_{y} \\
\sigma_{x y} \\
\sigma_{y z} \\
\sigma_{x z}
\end{array}\right\}=\left[\begin{array}{ccccc}
Q_{11} & Q_{12} & 0 & 0 & 0 \\
Q_{12} & Q_{22} & 0 & 0 & 0 \\
0 & 0 & Q_{66} & 0 & 0 \\
0 & 0 & 0 & Q_{44} & 0 \\
0 & 0 & 0 & 0 & Q_{55}
\end{array}\right]\left\{\begin{array}{l}
\varepsilon_{x} \\
\varepsilon_{y} \\
\gamma_{x y} \\
\gamma_{y z} \\
\gamma_{x z}
\end{array}\right\}
$$

where:

$$
\begin{aligned}
& Q_{11}=\frac{E_{1}}{1-v_{12} v_{21}} \\
& Q_{12}=\frac{v_{12} E_{2}}{1-v_{12} v_{21}}
\end{aligned}
$$

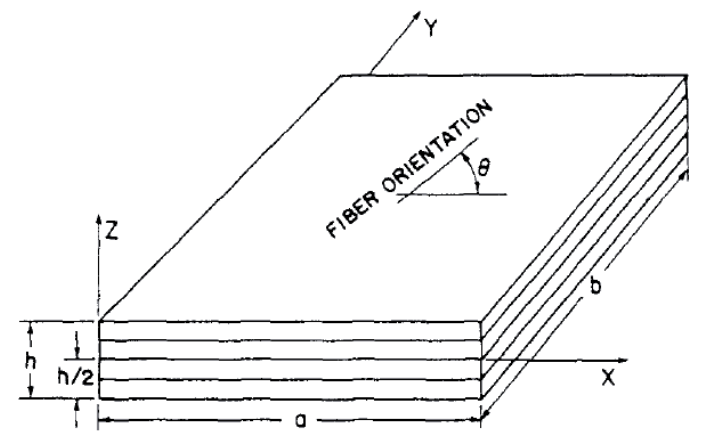

Fig. 1: Laminated plate nomenclature.

$Q_{22}=\frac{E_{2}}{1-v_{12} v_{21}}$

$Q_{66}=G_{12}, Q_{44}=G_{23}, Q_{55}=G_{13}$

Since the laminate is made of several orthotropic layers with their material axes oriented arbitrarily with respect to the laminate coordinates, the constitutive equations of each layer must be transformed to the laminate coordinates $(\mathrm{x}, \mathrm{y}, \mathrm{z})$. The stress-strain relations in the laminate coordinates of the kth layer are given as:

$\left\{\begin{array}{l}\sigma_{x} \\ \sigma_{y} \\ \sigma_{x y} \\ \sigma_{y z} \\ \sigma_{x z}\end{array}\right\}^{(k)}=\left[\begin{array}{ccccc}\bar{Q}_{11} & \bar{Q}_{12} & \bar{Q}_{16} & 0 & 0 \\ \bar{Q}_{12} & \bar{Q}_{22} & \bar{Q}_{26} & 0 & 0 \\ \bar{Q}_{16} & \bar{Q}_{26} & \bar{Q}_{66} & 0 & 0 \\ 0 & 0 & 0 & \bar{Q}_{44} & \bar{Q}_{45} \\ 0 & 0 & 0 & \bar{Q}_{45} & Q_{55}\end{array}\right\}^{(k)}\left\{\begin{array}{l}\varepsilon_{x} \\ \varepsilon_{y} \\ \gamma_{x y} \\ \gamma_{y z} \\ \gamma_{x z}\end{array}\right\}^{(k)}$

Where: $\bar{Q}_{i j}$ are the transformed material constants given as: 
$\bar{Q}_{11}=Q_{11} \cos ^{4} \theta+2\left(Q_{12}+2 Q_{66}\right) \sin ^{2} \theta \cos ^{2} \theta++Q_{22} \sin ^{4} \theta$

$\bar{Q}_{12}=\left(Q_{11}+Q_{22}-4 Q_{66}\right) \sin ^{2} \theta \cos ^{2} \theta+Q_{12}\left(\sin ^{4} \theta+\cos ^{4} \theta\right)$

$\bar{Q}_{22}=Q_{11} \sin ^{4} \theta+2\left(Q_{12}+2 Q_{66}\right) \sin ^{2} \theta \cos ^{2} \theta+Q_{22} \cos ^{4} \theta$

$\bar{Q}_{16}=\left(Q_{11}-Q_{12}-2 Q_{66}\right) \sin \theta \cos ^{3} \theta+\left(Q_{12}-Q_{22}+2 Q_{66}\right) \sin ^{3} \theta \cos \theta$

$\bar{Q}_{26}=\left(Q_{11}-Q_{12}-2 Q_{66}\right) \sin ^{3} \theta \cos \theta+\left(Q_{12}-Q_{22}+2 Q_{66}\right) \sin \theta \cos ^{3} \theta$

$\bar{Q}_{66}=\left(Q_{11}+Q_{22}-2 Q_{12}-2 Q_{66}\right) \sin ^{2} \theta \cos ^{2} \theta+Q_{66}\left(\sin ^{4} \theta+\cos ^{4} \theta\right)$

$\bar{Q}_{44}=Q_{44} \cos ^{2} \theta+Q_{55} \sin ^{2} \theta$

$\bar{Q}_{45}=\left(Q_{55}-Q_{44}\right) \cos \theta \sin \theta$

$\bar{Q}_{55}=Q_{55} \cos ^{2} \theta+Q_{44} \sin ^{2} \theta$

in which $\theta$ is the angle between the global $\mathrm{x}$-axis and the local $\mathrm{x}$-axis of each lamina.

\subsection{Equation of motions}

Denoting partial differentiation by a comma, the plate constitutive relations are of the form:

$$
\begin{aligned}
& \left\{\begin{array}{l}
M_{x} \\
M_{y} \\
M_{x y}
\end{array}\right\}=\left[\begin{array}{lll}
D_{11} & D_{12} & D_{16} \\
D_{12} & D_{22} & D_{26} \\
D_{16} & D_{26} & D_{66}
\end{array}\right]\left\{\begin{array}{c}
\theta_{x, x} \\
\theta_{y, y} \\
\theta_{y, x}+\theta_{x, y}
\end{array}\right\} \\
& \left\{\begin{array}{l}
Q_{y} \\
Q_{x}
\end{array}\right\}=k\left[\begin{array}{ll}
A_{44} & A_{45} \\
A_{45} & A_{55}
\end{array}\right]\left\{\begin{array}{l}
\theta_{y}+w_{0, y} \\
\theta_{x}+w_{0, x}
\end{array}\right\}
\end{aligned}
$$

Where: $\mathrm{k}$ is a shear correction factor as introduced by Mindlin. $\left(A_{i j,} D_{i j}\right)$ are the plate stiffness, defined by

$$
\begin{array}{ll}
D_{i j}=\int_{-h / 2}^{h / 2} \bar{Q}_{i j} z^{2} d z \quad(i, j=1,2,6) \\
A_{i j}=\int_{-h / 2}^{h / 2} \bar{Q}_{i j} d z \quad(i, j=4,5)
\end{array}
$$

Denoting normal stresses by $\sigma_{i}$ and shear stresses by $\tau_{i j}$, then

$$
\begin{aligned}
& \left(M_{x}, M_{y}, M_{x y}\right)=\int_{-h / 2}^{h / 2}\left(\sigma_{x}, \sigma_{y}, \sigma_{x y}\right) z d z= \\
& =\sum_{k=1}^{N} \int_{Z_{k}}^{Z_{k+1}}\left(\sigma_{x}, \sigma_{y}, \sigma_{x y}\right) z d z \\
& \left(Q_{x z}, Q_{y z}\right)=\int_{-h / 2}^{h / 2}\left(\sigma_{x z}, \sigma_{y z}\right) d z=\sum_{k=1}^{N} \int_{Z_{k}}^{Z_{k+1}}\left(\sigma_{x z}, \sigma_{y z}\right) d z
\end{aligned}
$$

Designating $\mathrm{C}_{1}$ as the straight configuration (impending state of buckling) and $\mathrm{C}_{2}$ as the bent configuration (just after buckling), the incremental loads $\Delta N_{x}$ and $\Delta N_{y}$ are equal to zero and the second Piola-Kirchhoff stress tensor at $\mathrm{C}_{1}$ is given by

$$
\begin{aligned}
\tau^{T} & =\left\{\begin{array}{lllll}
\sigma_{x} & \sigma_{y} & \tau_{x y} & \tau_{y z} & \tau_{z x}
\end{array}\right\} \\
& =\left\{\begin{array}{llllll}
-\hat{\sigma}_{x} & -\hat{\sigma}_{y} & 0 & 0 & 0
\end{array}\right\}
\end{aligned}
$$

Where: $\hat{\sigma}_{x}$ and $\hat{\sigma}_{y}$ consist of the stresses of different laminas, $\hat{\sigma}_{x}$ and $\hat{\sigma}_{y}(i=1,2,3 \ldots . ., l)$ which are related to the in-plane loads $N_{x}$ and $N_{y}$. The procedure for determining $\hat{\sigma}_{x}$ and $\hat{\sigma}_{y}$ will be given in due course.

Substituting eqns (3)-(13) into eqn (1) and then integrating with respect to $z$, the incremental total potential energy functional of a symmetric crossply laminated plate is derived as

$$
\Pi=\frac{1}{2} \int_{A}\left\{\begin{array}{l}
D_{11}\left(\frac{\partial \theta_{x}}{\partial x}\right)^{2}+D_{22}\left(\frac{\partial \theta_{y}}{\partial y}\right)^{2}+D_{66}\left(\frac{\partial \theta_{x}}{\partial y}+\frac{\partial \theta_{y}}{\partial x}\right)^{2}+2 D_{12} \frac{\partial \theta_{x}}{\partial x} \frac{\partial \theta_{y}}{\partial y}+A_{44}\left(\theta_{y}+\frac{\partial w_{0}}{\partial y}\right)^{2}+A_{55}\left(\theta_{x}+\frac{\partial w_{0}}{\partial x}\right)^{2} \\
-N_{x}\left(\frac{\partial w_{0}}{\partial x}\right)^{2}-N_{y}\left(\frac{\partial w_{0}}{\partial y}\right)^{2}-F_{x}\left[\left(\frac{\partial \theta_{x}}{\partial x}\right)^{2}+\left(\frac{\partial \theta_{y}}{\partial x}\right)^{2}\right]-F_{y}\left[\left(\frac{\partial \theta_{x}}{\partial y}\right)^{2}+\left(\frac{\partial \theta_{y}}{\partial y}\right)^{2}\right]
\end{array}\right\} d A
$$


In which the underlined terms are the so-called curvature terms which come from the higher order strain terms in eqn (4). The parameters $F_{x}$ and $F_{y}$ are determined by

$$
\left(F_{x}, F_{y}\right)=\int_{-h / 2}^{h / 2}\left(\hat{\sigma}_{x i}, \hat{\sigma}_{y i}\right) z^{2} d z
$$

$A$ is the plate area at the impending state of buckling $\mathrm{C}_{1}$, given by

$$
A=\left(a \Lambda_{x}\right)\left(b \Lambda_{y}\right)
$$

where: $\Lambda_{x}$ and $\Lambda_{y}$ are the pre-buckling in-plane deformation factors in the $\mathrm{x}$ and $\mathrm{y}$ directions, respectively.

When the plate is at the $\mathrm{C}_{1}$ configuration (impending buckling), the in-plane strains $\hat{\varepsilon}_{x}$ and $\hat{\varepsilon}_{y}$ can be obtained by:

$$
\left\{\begin{array}{l}
\hat{\varepsilon}_{x} \\
\hat{\varepsilon}_{y}
\end{array}\right\}=\left[\begin{array}{ll}
A_{11} & A_{12} \\
A_{21} & A_{22}
\end{array}\right]\left\{\begin{array}{l}
N_{x} \\
N_{y}
\end{array}\right\}
$$

where:

$$
A_{i j}=\int_{-h / 2}^{h / 2} \bar{Q}_{i j} d z \quad(i, j=1,2)
$$

The above equation implies that the strains $\hat{\varepsilon}_{x}$ and $\hat{\varepsilon}_{y}$ are identical in different laminas under in-plane loads $N_{x}$ and $N_{y}$ respectively. Thus, the prebuckling in-plane deformation factors $\Lambda_{x}$ and $\Lambda_{y}$ can be determined by
$\Lambda_{x}=1-\gamma \hat{\varepsilon}_{x}$

$\Lambda_{y}=1-\gamma \hat{\varepsilon}_{y}$

in which $\gamma$ is a scalar indicator. If $\gamma=1$, the effect of prebuckling in-plane deformation is considered; while if $\gamma=0$, this effect is ignored. Furthermore, the stresses $\hat{\sigma}_{x}$ and $\hat{\sigma}_{y}$ the ith lamina are given by

$\left\{\begin{array}{l}\hat{\sigma}_{x i} \\ \hat{\sigma}_{y i}\end{array}\right\}=\left[\begin{array}{ll}\bar{Q}_{11 i} & \bar{Q}_{12 i} \\ \bar{Q}_{21 i} & \bar{Q}_{22 i}\end{array}\right]\left\{\begin{array}{c}\hat{\varepsilon}_{x} \\ \hat{\varepsilon}_{y}\end{array}\right\}$

\section{Analytical Solutions for Symmetric Cross-ply Laminates}

For symmetric cross-ply laminates, the following plate stiffness's are identically zero:

$D_{16}=D_{26}=0$

For generality and convenience, the Cartesian coordinates (x, y) are non-dimensionalized as follows:

$\xi=\frac{x}{a \Lambda_{x}}$

$\eta=\frac{y}{b \Lambda_{y}}$

Substituting eqn (22) into eqn (14) leads to

$$
\begin{aligned}
& \pi=\frac{1}{2} \int_{0}^{1} \int_{0}^{1} \cdot\left\{D_{11}\left(\frac{1}{a \Lambda_{x}} \frac{\partial \theta_{x}}{\partial \xi}\right)^{2}+D_{22}\left(\frac{1}{b \Lambda_{y}} \frac{\partial \theta_{y}}{\partial \eta}\right)^{2}+D_{66}\left(\frac{1}{b \Lambda_{y}} \frac{\partial \theta}{\partial \eta}+\frac{1}{a \Lambda_{x}} \frac{\partial \theta_{x}}{\partial \eta}\right)^{2}+2 D_{12} \frac{1}{a b \Lambda_{x} \Lambda_{y}} \frac{\partial \theta_{x}}{\partial \xi} \frac{\partial \theta_{y}}{\partial \eta}\right. \\
& +A_{44}\left(\theta_{y}+\frac{1}{b \Lambda_{y}} \frac{\partial w_{0}}{\partial \eta}\right)^{2}+A_{55}\left(\theta_{x}+\frac{1}{a \Lambda_{x}} \frac{\partial w_{0}}{\partial \xi}\right)^{2}-N_{0}\left[\left(\frac{1}{a \Lambda_{x}} \frac{\partial w_{0}}{\partial \xi}\right)^{2}+\beta\left(\frac{1}{b \Lambda_{y}} \frac{\partial w_{0}}{\partial \eta}\right)^{2}\right] \\
& \left.-\mu F_{x}\left[\left(\frac{1}{a \Lambda_{x}} \frac{\partial w_{0}}{\partial \xi}\right)^{2}+\beta\left(\frac{1}{b \Lambda_{y}} \frac{\partial w_{0}}{\partial \eta}\right)^{2}\right]-\mu F_{y}\left[\left(\frac{1}{a \Lambda_{x}} \frac{\partial w_{0}}{\partial \xi}\right)^{2}+\beta\left(\frac{1}{b \Lambda_{y}} \frac{\partial w_{0}}{\partial \eta}\right)^{2}\right]\right\} a b \Lambda_{x} \Lambda_{y} d \xi d \eta
\end{aligned}
$$


A scalar indicator $\mu$ is introduced in the above equation. If $\mu=1$, the higher-order terms (curvature terms) are included in the energy functional. If $\mu=0$, the curvature terms are neglected.

Taking the stationary conditions of eqn (23) with respect to $w_{0}, \theta_{x}$ and $\theta_{y}$ using the Euler-Lagrange equation yields the following governing differential equations for buckling of symmetric cross-ply laminated plates with allowance for prebuckling in-plane deformation and curvature terms:

$$
\begin{aligned}
& \frac{1}{\partial \xi}\left[\frac{1}{a^{2} \Lambda_{x}^{2}}\left(D_{11}-\mu F_{x}\right) \frac{\partial \theta_{x}}{\partial \xi}+\frac{D_{12}}{a b \Lambda_{x} \Lambda_{y}} \frac{\partial \theta_{y}}{\partial \eta}\right]+\frac{1}{\partial \eta}\left[\frac{1}{b^{2} \Lambda_{y}^{2}}\left(D_{66}-\mu F_{y}\right) \frac{\partial \theta_{x}}{\partial \eta}+\frac{D_{66}}{a b \Lambda_{x} \Lambda_{y}} \frac{\partial \theta_{y}}{\partial \xi}\right] \\
& -A_{55}\left(\theta_{x}+\frac{1}{a \Lambda_{x}} \frac{\partial w_{0}}{\partial \xi}\right)=0
\end{aligned}
$$

$$
\begin{aligned}
& \frac{1}{\partial \xi}\left[\frac{1}{a^{2} \Lambda_{x}^{2}}\left(D_{66}-\mu F_{x}\right) \frac{\partial \theta_{x}}{\partial \xi}+\frac{D_{66}}{a b \Lambda_{x} \Lambda_{y}} \frac{\partial \theta_{x}}{\partial \eta}\right]+\frac{1}{\partial \eta}\left[\frac{1}{b^{2} \Lambda_{y}^{2}}\left(D_{22}-\mu F_{y}\right) \frac{\partial \theta_{y}}{\partial \eta}+\frac{D_{12}}{a b \Lambda_{x} \Lambda_{y}} \frac{\partial \theta_{y}}{\partial \xi}\right] \\
& -A_{44}\left(\theta_{y}+\frac{1}{a \Lambda_{y}} \frac{\partial w_{0}}{\partial \eta}\right)=0
\end{aligned}
$$

$$
\begin{aligned}
& \frac{A_{55}}{a \Lambda_{x}} \frac{\partial}{\partial \xi}\left(\theta_{x}+\frac{1}{a \Lambda_{x}} \frac{\partial w_{0}}{\partial \xi}\right)+\frac{A_{44}}{b \Lambda_{y}} \frac{\partial}{\partial \eta}\left(\theta_{y}+\frac{1}{b \Lambda_{y}} \frac{\partial w_{0}}{\partial \eta}\right)- \\
& -N_{0}\left[\frac{1}{a^{2} \Lambda_{x}^{2}} \frac{\partial}{\partial \xi}\left(\frac{\partial w_{0}}{\partial \xi}\right)+\frac{\beta}{b^{2} \Lambda_{y}^{2}} \frac{\partial}{\partial \eta}\left(\frac{\partial w_{0}}{\partial \eta}\right)\right]=0 \quad \text { Where: }
\end{aligned}
$$

\section{Where:}

Let the origin of coordinates $(\xi, \eta)$ be at the bottom left corner of the plate. The trigonometric functions

$$
\begin{aligned}
& \theta_{x}(\xi, \eta)=A_{m n} \cos (m \pi \xi) \sin (n \pi \eta) \\
& \theta_{y}(\xi, \eta)=B_{m n} \sin (m \pi \xi) \cos (n \pi \eta) \\
& w_{0}(\xi, \eta)=C_{m n} \sin (m \pi \xi) \sin (n \pi \eta)
\end{aligned}
$$

Substituting eqn(27) into the differential equations [eqns(24)-(26)] the governing eigenvalue equation, which determines the buckling load of the simply supported rectangular plate, is derived as

$$
\left[\begin{array}{ccc}
p_{1} & p_{2} & p_{3} \\
p_{2} & p_{4} & p_{5} \\
p_{3} & p_{5} & p_{6}-N_{0} p_{7}
\end{array}\right]\left\{\begin{array}{l}
A_{m n} \\
B_{m n} \\
C_{m n}
\end{array}\right\}=\left\{\begin{array}{l}
0 \\
0 \\
0
\end{array}\right\}
$$

$$
\begin{aligned}
& p_{1}=\pi^{2}\left(\frac{D_{11} m^{2}}{a^{2} \Lambda_{x}^{2}}+\frac{D_{66} n^{2}}{b^{2} \Lambda_{y}^{2}}\right)+A_{55}- \\
& -\mu \pi^{2}\left(F_{x} \frac{m^{2}}{a^{2} \Lambda_{x}^{2}}+F_{y} \frac{n^{2}}{b^{2} \Lambda_{y}^{2}}\right), \\
& p_{2}=\left(D_{11}+D_{66}\right) \frac{m n \pi^{2}}{a b \Lambda_{x} \Lambda_{y}}, \quad p_{3}=\frac{m \pi}{a \Lambda_{x}} \\
& p_{4}=\pi^{2}\left(\frac{D_{66} m^{2}}{a^{2} \Lambda_{x}^{2}}+\frac{D_{22} n^{2}}{b^{2} \Lambda_{y}^{2}}\right)+A_{44}- \\
& -\mu \pi^{2}\left(F_{x} \frac{m^{2}}{a^{2} \Lambda_{x}^{2}}+F_{y} \frac{n^{2}}{b^{2} \Lambda_{y}^{2}}\right), \\
& p_{5}=\frac{n \pi}{b \Lambda_{y}}, \quad p_{6}=\pi^{2}\left(\frac{A_{55} m^{2}}{a^{2} \Lambda_{x}^{2}}+\frac{A_{44} n^{2}}{b^{2} \Lambda_{y}^{2}}\right), \\
& p_{7}=\pi^{2}\left(\frac{m^{2}}{a^{2} \Lambda_{x}^{2}}+\frac{n^{2}}{b^{2} \Lambda_{y}^{2}}\right)
\end{aligned}
$$


For nontrivial solution of the buckling load $N_{0}$ the determinant of the matrix in eqn (28) must be equal to zero. It is seen from eqns (17) and (19) that the pre-buckling in-plane deformation factors $\Lambda_{x}$ and $\Lambda_{y}$ are functions of in-plane load $N_{0}$. Consequently, the terms $\mathrm{Pi}_{\mathrm{i}}, \mathrm{i}=1,2 \ldots, 7$ in eqn (29) are all functions of $N_{0}$ and the determinant of the matrix in eqn (28) is a high-order function of . In view of the difficulty involved in a continued analytical development, a numerical procedure is implemented to obtain closed-form solutions for the buckling load $N_{0}$.

The influence of load $N_{0}$ in $\mathrm{Pi}_{\mathrm{i}} \mathrm{i}=1-7$, is revealed through the prebuckling in-plane deformation terms $\Lambda_{x}$ and $\Lambda_{y}$ and the so-called curvature terms (when $\mu=1$ ). Viewing eqn (28), the buckling load $N_{0}$ can be expressed as

$$
N_{0}=\frac{P_{3}^{2} P_{4}-2 P_{2} P_{3} P_{5}+P_{1} P_{5}^{2}+P_{2}^{2} P_{6}-P_{1} P_{4} P_{6}}{P_{7}\left(P_{2}^{2} P_{6}-P_{1} P_{4}\right)}
$$

\section{Numerical Results and Discussion}

In this section, various numerical examples are described and discussed for verifying the accuracy of the present method in predicting the buckling behaviors of simply supported symmetric crossply laminates. For the verification purpose, the results obtained by present method are compared with those of the results of previous works in the literature and computer code Ansys. In all examples, a shear correction factor of $5 / 6$ is used for FSDT. The following lamina propertied is used: Material $[19,20,21]$

$$
E_{1} / E_{2}=\text { open, } E_{2}=E_{3} \text {, }
$$

$G_{12}=G_{13}=0.6 E_{2}, G_{23}=0.5 E_{2}$,

$v_{12}=v_{13}=0.25, v_{23}=0.49$

The material properties are assumed to be the same for all layers and the critical buckling loads are normalized as

$\bar{N}=\frac{N_{c r} a^{2}}{E_{2} h^{3}}$

In order to verify the present solutions, the convergence properties of the biaxial critical buckling loads of square cross-ply laminated composite plates are presented in Table 1. As table shows, the present results have a good agreement with Refs. $[19,20,21]$ and finite element method using Ansys.

Table 2 presents a comparison of the lowest critical buckling loads of three-layer cross-ply laminated plates with analytical solutions $[20,21]$ and finite element method for various values of the degree of orthotropy of the individual layers $\mathrm{E}_{1} / \mathrm{E}_{2}$. They are in excellent agreement.

Figures 2-6 display the first mode shapes of a symmetric cross-ply laminated square plates $\left(0^{\circ} / 90^{\circ} / 0^{\circ} ; \mathrm{a} / \mathrm{h}=10\right)$ for $\mathrm{E} 1 / \mathrm{E} 2=2,10,20,30,40$ respectively with simply supported (elements Shell 99). The value of the nondimensional critical buckling load of the graphs of first mode exists in table 1 .

The effects of aspect ratio $a / b$ on nondimensional critical buckling load of simply supported symmetric cross-ply rectangular laminates under uniaxial compression, compression along the $x$-axis and tension along the $y$-axis

Table 2: Comparison of lowest biaxial critical buckling loads ( $a / h=10)$ Comparison of buckling factors, $\bar{N}=N_{c r} a^{2} / E_{2} h^{3}$, for simply supported symmetric cross-ply laminated square plates $a / h=10$, three-ply) subject to biaxial compression $\left(N_{x}=N_{y}=N_{0}\right)$ without consideration

\begin{tabular}{|c|c|c|c|c|c|c|c|}
\hline$E_{1} / E_{2}$ & Present FSDT & Present Ansys & СLHOT [21] & HSDT [20] & HSDT [19] & FSDT [20] & CPT [20] \\
\hline 2 & 2.3442 & 2.1277 & 2.36625 & 2.343 & 2.364 & 2.344 & 2.473 \\
\hline 10 & 4.9355 & 4.7510 & 4.96038 & 4.916 & 4.963 & 4.936 & 5.746 \\
\hline $20^{\mathrm{a}}$ & 7.4923 & 7.3350 & 7.49323 & 7.449 & 5.516 & 7.588 & 9.591 \\
\hline $30^{a}$ & 8.9751 & 9.3757 & 8.80314 & 8.820 & 9.056 & 8.575 & 12.147 \\
\hline $40^{\mathrm{a}}$ & 10.2024 & 11.0350 & 9.82430 & 9.975 & 10.259 & 10.202 & 14.704 \\
\hline
\end{tabular}
of the effects of prebuckling in-plane deformation and curvature terms.

${ }^{a}$ The lowest critical buckling occurs at mode numbers $m=1, n=2$. 


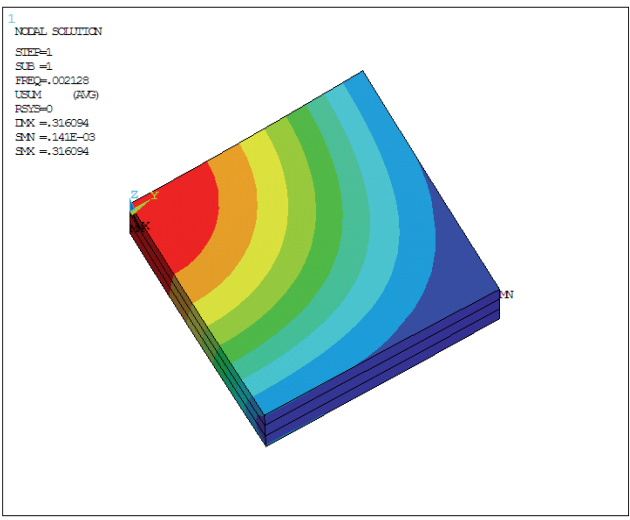

Fig. 2: First buckling mode of symmetric cross-ply laminated square plates subjected to biaxial compression $\left(0^{\circ} \% 0^{\circ} / 0^{\circ} ; a / h=10\right.$, $\left.E_{1} / E_{2}=2\right)$, Shelg9 element.

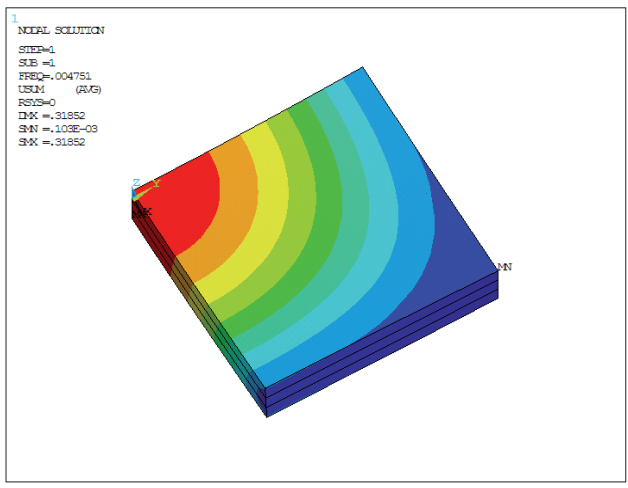

Fig. 3: First buckling mode of symmetric cross-ply laminated square plates subjected to biaxial compression $\left(0^{\circ} / 90^{\circ} \% 0^{\circ} ; \mathrm{a} / \mathrm{h}=10\right.$, $\left.E_{1} / E_{2}=10\right)$, Shelg9 element.

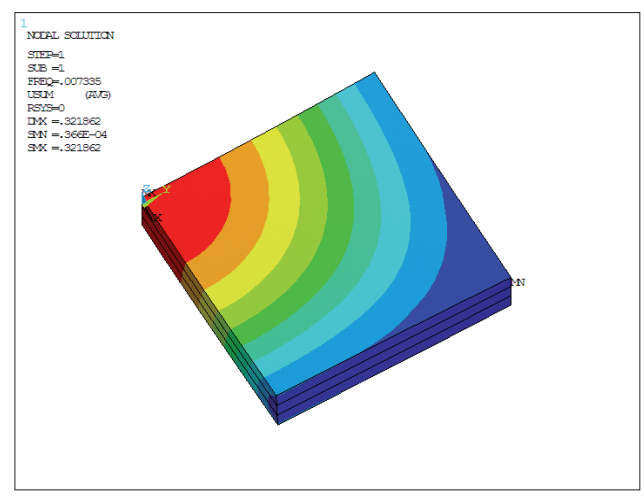

Fig. 4: First buckling mode of symmetric cross-ply laminated square plates subjected to biaxial compression $\left(0^{\circ} / 90^{\circ} \% ; a / h=10\right.$, $\left.E_{1} / E_{2}=20\right)$, Shelg9 element.

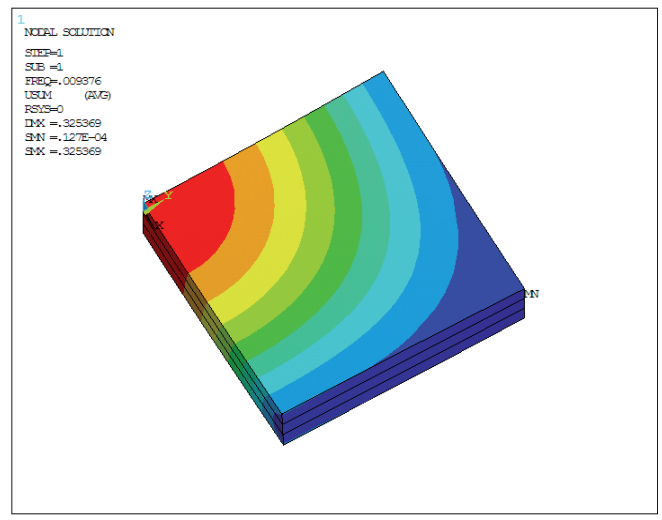

Fig. 5: First buckling mode of symmetric cross-ply laminated square plates subjected to biaxial compression $\left(0^{\circ} / 90^{\circ} \% 0^{\circ} ; \mathrm{a} / \mathrm{h}=10\right.$, $E_{1} / E_{2}=30$ ), Shelg9 element.

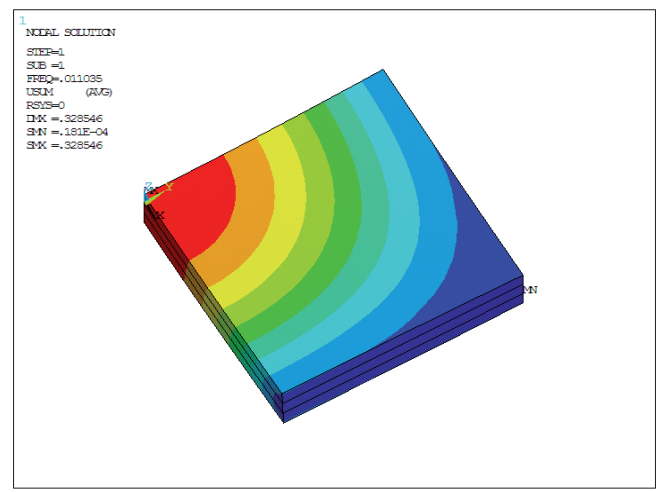

Fig. 6: First buckling mode of symmetric cross-ply laminated square plates subjected to biaxial compression $10^{\circ} / 90^{\circ} \% 0^{\circ} ; a / h=10$, $\left.E_{1} / E_{2}=40\right)$, Shel99 element.

Table 2: Comparison of non-dimensional biaxial critical buckling loads $\left(a / h=10, E_{1} / E_{2}=40\right)$, for simply supported symmetric crossply $0 \% 90 \% 0^{\circ}$ without consideration of the effects of prebuckling in-plane deformation and curvature terms.

\begin{tabular}{|l|l|l|l|l|}
\hline $\mathbf{m}, \mathbf{n}$ & $\begin{array}{l}\text { Present } \\
\text { FSDT }\end{array}$ & $\begin{array}{l}\text { Present } \\
\text { Ansys }\end{array}$ & \multicolumn{1}{l|}{$\begin{array}{l}\text { GLHOT } \\
\text { [21] }\end{array}$} & \multicolumn{1}{l|}{ HSDT [20] } \\
\hline 1,1 & 11.1576 & 11.0350 & 11.08254 & 11.060 \\
\hline 1,2 & 10.2024 & 14.2070 & 9.82430 & 9.975 \\
\hline 1,3 & 14.3344 & 23.9280 & 13.05053 & 13.627 \\
\hline 1,5 & 23.9730 & 29.2210 & 20.83553 & 21.795 \\
\hline 1,7 & 30.7596 & 32.9530 & 27.63812 & 27.465 \\
\hline 1,9 & 34.9727 & 35.5320 & 30.52917 & 31.280 \\
\hline
\end{tabular}




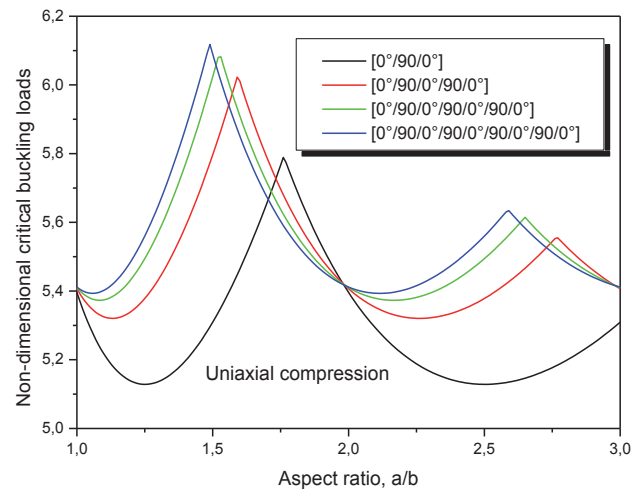

Fig. 7: Variation of the non-dimensional critical buckling loads versus the aspect ratio $a / b$ of plates for simply supported symmetric cross-ply rectangular laminates subject to uniaxial compression $(\gamma=0, \mu=0)$.

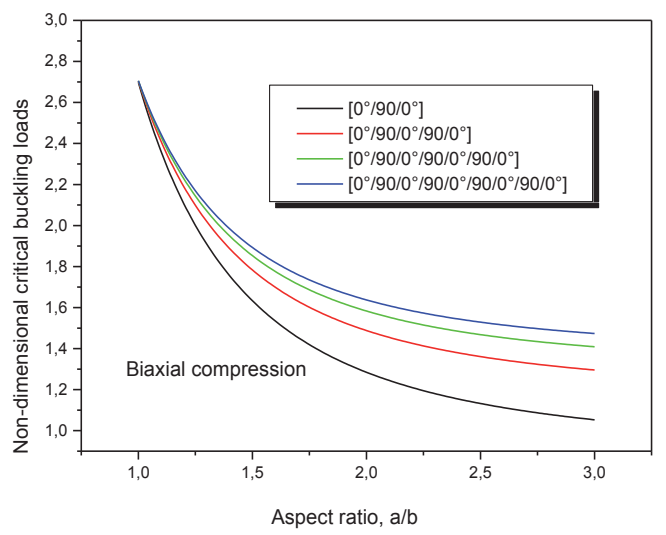

Fig. 8: Variation of the non-dimensional critical buckling loads versus the aspect ratio a/b of plates for simply supported symmetric cross-ply rectangular laminates subject to biaxial compression $(\gamma=0, \mu=0)$.

and biaxial compression are shown in Figs. 7 and 8 , respectively. The thickness ratio $\mathrm{b} / \mathrm{h}$ is assumed to be 10 for stacking sequences of $\left[0^{\circ} / 90 / 0^{\circ}\right],\left[0^{\circ} / 90 / 0^{\circ} / 90 / 0^{\circ}\right]$, $\left[0^{\circ} / 90 / 0^{\circ} / 90 / 0^{\circ} / 90 / 0^{\circ}\right]$, $\left[0^{\circ} / 90 / 0^{\circ} / 90 / 0^{\circ} / 90 / 0^{\circ} / 90 / 0^{\circ}\right]$. It is shown that the nondimensional critical buckling load generally decreases by the increase of the aspect ratio a/b. In the case of uniaxial compression as shown in Fig. 7, the graphs are not smooth due to the change of critical buckling mode as the aspect ratio increases. Whereas, the graph in the case of biaxial compression as shown in Fig. 8 is smooth due to the existence of a single critical buckling mode regardless of aspect ratio a/b. On the other hand, the nondimensional critical buckling load increases, when the number of plies is increased.

A comparison of Fig. 7 with Fig. 8 shows that the nondimensional critical buckling load for the plate under uniaxial compression $\left(N_{x}=N_{0}, N_{y}=0\right)$ is greater than that under biaxial compression $\left(N_{x}=\right.$ $\left.N_{y}=N_{0}\right)$.

\section{Conclusions}

An exact solution has been developed to investigate the buckling behavior of simply supported symmetrical cross-ply rectangular plates subjected to uniaxial compression and biaxial compression. The present exact solution is developed on the basis of the first-order shear deformation lamination theory. The results of the present solution are verified by with existing results in the literature for some special cases and computer code Ansys. A parametric study is conducted to investigate the effects of aspect ratio, thickness-to-width ratio and the modulus ratio on the buckling load factor. Based on the results discussed earlier, major conclusions can be summarized as follows:

1. It is observed that as the aspect ratio a/b increases, the critical buckling load mainly decrease.

2. The numbers of layer significantly affect the buckling coefficient. 3. The increasing layer number increases the critical buckling load. 4. The buckling load of the plate under uniaxial compression is greater than the one under biaxial compression.

\section{References}

[1] G.J.Turvey, I.H. Marshall, Buckling and Postbuckling of Composite Plates, Chapman and Hall, London, 1995, p.396.

[2] Pagano NJ. Exact solution for rectangular bi-directional composites and sandwich plates. J Compos Mater 1970;4:20-34.

[3] Noor AK. Stability of multilayered composite plates. Fiber Sci Tech 1975;8(2):81-9.

[4] Noor AK. Free vibration of multilayered composite plates. AIAA J 1973;11:1038-9.

[5] Marshell, I.H., Rhodes, J., Banks, W.M.. The nonlinear behaviour of thin orthotopic, curved panels under lateral loading. Journal of Mechanical Engineering Science, 1977,19 (1), 30-37.

[6] Kim, K., Voyiadjis, G.Z. Nonlinear finite element of composite panels. Composites Part B: Engineering , 1999, 30, 365-381.

[7] J.H. Kang, A.W. Leissa, Exact solutions for the buckling of rectangular plates having linearly varying in-plane loading on two opposite simply supported edges, International 
Journal of Solids and Structures 42 (2005) 4220-4238.

[8] J.N. Reddy, N.D. Phan, Stability and vibration of isotropic, orthotropic and laminated plates according to a higher-order shear deformation theory, Journal of Sound and Vibration 98 (1985) 157-170.

[9] M. Bouazza, A. Tounsi, E.A. Adda-Bedia, A. Megueni, Thermal buckling of simply supported FGM square plates, Applied Mechanics and Materials 61 (2011) 25-32.

[10] M. Bouazza, E.A. Adda-Bedia, Elastic stability of functionally graded rectangular plates under mechanical and thermal loadings, Academic Journals, Scientific Research and Essays, Vol. 8(39), pp. 1933-1943, 2013.

[11] M. Bouazza, A. Tounsi, E.A. Adda-Bedia, A. Megueni, Thermoelastic stability analysis of functionally graded plates: An analytical approach, Computational Materials Science 49 (2010) 865-870.

[12] M. Bouazza, A. Tounsi, E.A. Adda-Bedia, A. Megueni, Stability analysis of functionally graded plates subject to thermal loads, Shell-like Structures-Advanced Structured Materials, Springer 15 (8) (2011) 669-680.

[13] H. Matsunaga, Buckling Instabilities of of Thick Elastic Plates Subjected to In-Plane Stresses, Computers \& Structures, vol. 62, no. 1, pp. 205-214, 1995.

[14] H. Matsunaga, Vibration and Stability of Cross-Ply Laminated Composite Plates According to a Global High-Order Plate Theory, Computers \& Structures, vol. 48, pp. 231-244, 2000.

[15] A. K. Noor and P. M. Burton, Finite Element Buckling and Post-Buckling Solutions for Multilayered Composite Plates. Finite Elements in Analysis and Design, vol. 15, pp. 343-367, 1994.

[16] A. J. M. Ferreira and J. T. Barbosa, Buckling Behaviour of Composite Shells, Composite Structures, vol. 50, no. 1, pp. 93-98, 2000.

[17] A. J. M. Ferreira, J. E. Fassheur, and R. C. Batra, Natural Frequencies of Thick Plate Made of Orthotropic, Monoclinic and Hexagonal Materials by a Meshless Method, Journal of Sound and Vibration, vol. 319, nos. 3-5, pp. 984-992, 2009.

[18] Washizu, K. (1975). Variational Methods in Elasticity and Plasticity (2nd edn), Pergamon Press, Oxford.

[19] Khdeir AA, Librescu L. Analysis of symmetric cross-ply laminated elastic plates using a higher-order theory. Part ॥-buckling and free vibration. Composite Structures 1988;9:259-77.

[20] Fares ME, Zenkour AM. Buckling and free vibration of nonhomogeneous composite cross-ply laminated plates with various plate theories. Composite Structures 1999; 44:27987.

[21] Zhen Wu, Wanji Chen. Thermomechanical buckling of laminated composite and sandwich plates using global-local higher order theory. International Journal of Mechanical Sciences 49 (2007) 712-721 